\title{
ASOCIACIONES DE OSTRÁCODOS BENTÓNICOS ACTUALES Y DEL HOLOCENO DEL ESTUARIO DE BAHÍA BLANCA (BUENOS AIRES, ARGENTINA): INTERPRETACIONES PALEOAMBIENTALES
}

\author{
ROMINA GISELA KIHN \\ Instituto de ciencias de la tierra y ambientales de La Pampa, CONICET, Mendoza 109, Santa Rosa 6300, \\ La Pampa, Argentina.rgkihn@gmail.com \\ DINA E. MARTÍNEZ \\ Departamento de Geología, Universidad Nacional del Sur, San Juan 670, Bahía Blanca 8000, Buenos Aires, \\ Argentina.dinamart@criba.edu.ar \\ EDUARDO A. GÓMEZ \\ Instituto Argentino de Oceanografía, CCT CONICET Bahía Blanca, Florida 75000; 8000, UTN FRBB, \\ 11 de Abril 461, B8000LMI Bahía Blanca, Buenos Aires, Argentina.gmgomez@criba.edu.ar \\ CLAUDIA M. BOREL \\ INGEOSUR. San Juan 670, B8000ICN Bahía Blanca, Argentina.maborel@criba.edu.ar
}

\begin{abstract}
RECENT AND HOLOCENE BENTHIC OSTRACOD ASSEMBLAGES OF THE BAHIA BLANCA ESTUARY: (BUENOS AIRES, ARGENTINA): PALAEOENVIRONMENTAL INTERPRETATIONS. The study of ostracod fauna in sediment samples from present environments at the Bahía Blanca estuary led to the identification of different ostracod associations, through which it was possible to identify Holocene depositional environments. Based on the systematic analysis of the ostracods present in different core levels, a total of 11 genera represented by 13 species of benthic ostracods were identified, out of which Neocytherideis ruidis, Loxocythere variasculpta and Callistocythere litoralensis were dominant along the core. The quantitative and qualitative analysis of ostracods from the Holocene allowed us to determine the following palaeoenvironments: at the base of the core the sediments were deposited in an intertidal environment with developed mudflats; the middle sector evidenced an increase in environmental energy; and the upper portion of the core contained deposits of marshes environment with greater freshwater input. The detailed study of the benthic Ostracoda allowed to identify palaeoenvironments and to provide new evidence of negative fluctuations of the mean sea level for the Holocene.
\end{abstract}

Key words: ostracods, estuary, Bahía Blanca, palaeoenvironment, Argentina.

RESUMO - O estudo da fauna presente nas amostras de sedimento de ambientes atuais do Estuário de Bahía Blanca permitiu a identificação de diferentes associações de ostracodes que facilitaram o reconhecimento dos ambientes deposicionais do Holoceno. A partir da análise sistemática da ostracofauna encontrada nos diferentes níveis do testemunho, foi identificado um total de 11 gêneros representados por 13 espécies de ostracodes bentônicos, entre as quais houve um predomínio de Neocytherideis ruidis, Loxocythere vasriasculpta e Callistocythere litoralensis ao longo de todo o testemunho. A análise quantitativa e qualitativa da ostracofauna do Holoceno permitiu determinar os seguintes paleoambientes: na base do testemunho os sedimentos foram depositados em uma zona entremarés com marismas desenvolvidas; na parte média observou-se um aumento na energia ambiental e no topo do testemunho foram registrados depósitos de um ambiente de marismas com maior presença de águas continentais. O estudo detalhado dos ostracodes bentônicos permitiu identificar paleoambientes e fornecer novas evidências de flutuações negativas do nível médio do mar para o Holoceno.

Palavras-chave: ostracodes, estuário, Bahía Blanca, paleoambiente, Argentina.

\section{INTRODUCCIÓN}

El estudio micropaleontológico de sedimentos recientes es una técnica muy utilizada en los últimos años, ya que proporciona datos directamente aplicables en las reconstrucciones paleoambientales. Los ostrácodos son microcrustáceos ampliamente utilizados para la caracterización de los distintos ambientes del pasado; poseen un caparazón calcáreo compuesto por dos valvas, son fundamentalmente bentónicos, con un amplio espectro ecológico; habitan ambientes de agua dulce, salobre, marina e hipersalino. Son importantes indicadores paleoecológicos y paleoestratigráficos, dado que son muy sensibles a los cambios ecológicos y presentan un rango estratigráfico muy 
amplio, debido a que se registran a partir del Ordovícico hasta la actualidad (Horne et al., 2002; Laprida, 2006; Laprida \& Ballent, 2007).

El conocimiento de las preferencias y limitaciones ecológicas de las especies de ostrácodos, permite conocer la evolución de los principales parámetros ambientales desde medios del pasado hasta la actualidad. Por ello cobra especial interés el estudio del mayor número posible de especies actuales, su distribución y su caracterización ecológica.

Las áreas litorales constituyen ecosistemas muy dinámicos y a menudo influenciados por la actividad humana. Es por ello que el estudio de las asociaciones de ostrácodos de estas áreas resulta de gran interés, dado que posibilita la caracterización de episodios de evolución natural y otros de influencia antrópica en estos ambientes.

Hasta el momento, son escasos los estudios realizados sobre la ostracofauna presente en el área de Bahía Blanca; podemos mencionar los trabajos realizados por Bertels \& Martínez (1990, 1997), Bertels-Psotka \& Martínez (1999) y Martínez (2005). En áreas próximas al río Quequén Grande es importante el aporte de Ferrero $(2006,2009)$ más hacia el norte, en la cuenca del Salado, Bertels-Psotka \& Laprida (1998a,b,c,d) realizaron estudios sistemáticos e interpretaciones paleoecológicas de los ostrácodos límnicos, transicionales y marinos.

El objetivo de este trabajo es contribuir con nueva información micropaleontológica a fin de caracterizar los paleoambientes desarrollados durante el Holoceno en el estuario de Bahía Blanca, sobre la base de estudios cualitativos y cuantitativos de ostrácodos bentónicos.

\section{ÁREA DE ESTUDIO}

El estuario de Bahía Blanca está ubicado en el extremo sur de la provincia de Buenos Aires, República Argentina. Es un sistema mesomareal conformado por canales de diversas dimensiones y orientados en dirección Noroeste-Sureste. Estos canales poseen cauces sinusoidales, separados por islas y extensas llanuras de marea compuestas en general por sedimentos limoarcillosos que se encuentran en constante evolución morfodinámica (Piccolo et al., 1987). Los aportes de agua dulce provienen del rio Sauce Chico y del arroyo Napostá Grande, los cuales contribuyen con bajos volúmenes de agua y sedimentos (Ginsberg \& Perillo, 1990).

Las islas están compuestas por comunidades vegetales típicas de la estepa halófila (Verettoni, 1961; Verettoni \& Aramayo, 1976). Las planicies de marea en las áreas topográficamente más altas están cubiertas por una densa vegetación de Spartina, mientras que en las áreas más bajas no hay cobertura vegetal.

Las salinidades son verticalmente homogéneas, excepto en los sectores asociados a la desembocadura de arroyos donde el agua presenta una estratificación marcada (Piccolo et al., 1987). La distribución longitudinal de la salinidad en el canal principal presenta un patrón homogéneo, salvo en la cabecera del estuario donde durante el invierno debido a la mayor descarga fluvial se registran las concentraciones de salinidades más bajas (25 a 30 ups), pudiendo llegar a valores de 15 durante períodos lluviosos (Piccolo et al., 1987), mientras que en el verano por evaporación se dan las máximas salinidades habiéndose registrado incluso valores excepcionalmente altos (52 ups) durante el mes de enero (Freije et al., 1981).

Freije et al. (1981) consideran que el proceso de evaporación (favorecido por la influencia de vientos continentales secos que soplan sobre las planicies de sal) es el principal responsable de la hipersalinidad producida en el área interna (Beigt, 2007). Dentro del Canal Principal se encuentra el canal Tres Brazas, que es uno de los más largos que desembocan en el canal anteriormente mencionado. El canal Tres Brazas no presenta descarga de agua dulce y su circulación es totalmente dominada por las mareas. Este canal es altamente sinuoso y posee numerosos canales tributarios que drenan las planicies de marea, entre ellos tenemos el canal Tierra Firme (Ginsberg \& Perillo, 1990).

\section{MATERIAL Y MÉTODOS}

\section{Sedimentos actuales}

En este trabajo se seleccionaron cuatro zonas de muestreo que abarcan una marcada variabilidad de condiciones ambientales, tanto en función de sus características hidrológicas y sedimentarias, como del ambiente biológico, caracterizado por el tipo de vegetación dominante. Los sitios muestreados fueron (Figura 1): (i) balneario Villa del Mar (V) (38 $52^{\prime}$ S- $\left.62^{\circ} 06^{\prime} \mathrm{O}\right)$ que se encuentra en el sector externo del Canal Principal, en la transición entre los ambientes dominados por sedimentos finos y las costas arenosas. El patrón de zonación de la vegetación típico de esta zona del estuario incluye marismas de Spartina alterniflora, marismas de Sarcocornia perennis y marismas mixtas de Spartina perennis y $S$. densiflora; (ii) Club Almirante Brown (B) (38 $8^{\circ} 45^{\prime} \mathrm{S}-62^{\circ} 18^{\prime} \mathrm{O}$ ubicado en el sector interno del Canal Principal, se caracteriza por presentar el patrón de zonación típico de la cabecera del estuario, con ausencia de vegetación en la zona intermareal y marismas de S. perennis colonizando elevaciones por encima del nivel de las mareas ordinarias; (iii) boca del Canal Maldonado (M) $\left(38^{\circ} 44^{\prime} \mathrm{S}-62^{\circ} 19^{\prime} \mathrm{O}\right)$ que presenta la particularidad de que allí crece la única marisma de Spartina alterniflora de la zona interna del estuario; y (iv) planicies y marismas vegetadas por Spartina alterniflora del Canal Tres Brazas (CTB) $\left(38^{\circ} 55^{\prime} \mathrm{S}-62^{\circ} 14^{\prime} \mathrm{O}\right)$, ubicado en la zona media del estuario de Bahía Blanca. Este canal no presenta descarga de agua dulce, razón por la que su circulación es totalmente mareal dominante. Se caracteriza por ser altamente sinuoso y por poseer numerosos canales tributarios que drenan las planicies de marea; entre ellos está Tierra Firme que se une al canal Tres Brazas, aproximadamente a 2,5 km de su desembocadura (Ginsberg \& Perillo, 1990).

\section{Sedimentos del Holoceno}

Se estudió un testigo obtenido en el canal Bahía del Palo ( $38^{\circ} 53^{\prime} \mathrm{S}-62^{\circ} 14^{\prime} \mathrm{O}$ ), tributario del canal Tierra Firme, el cual a su vez es tributario del canal Tres Brazas, que desemboca en el Canal Principal del Estuario de Bahía Blanca (Figura 1). 


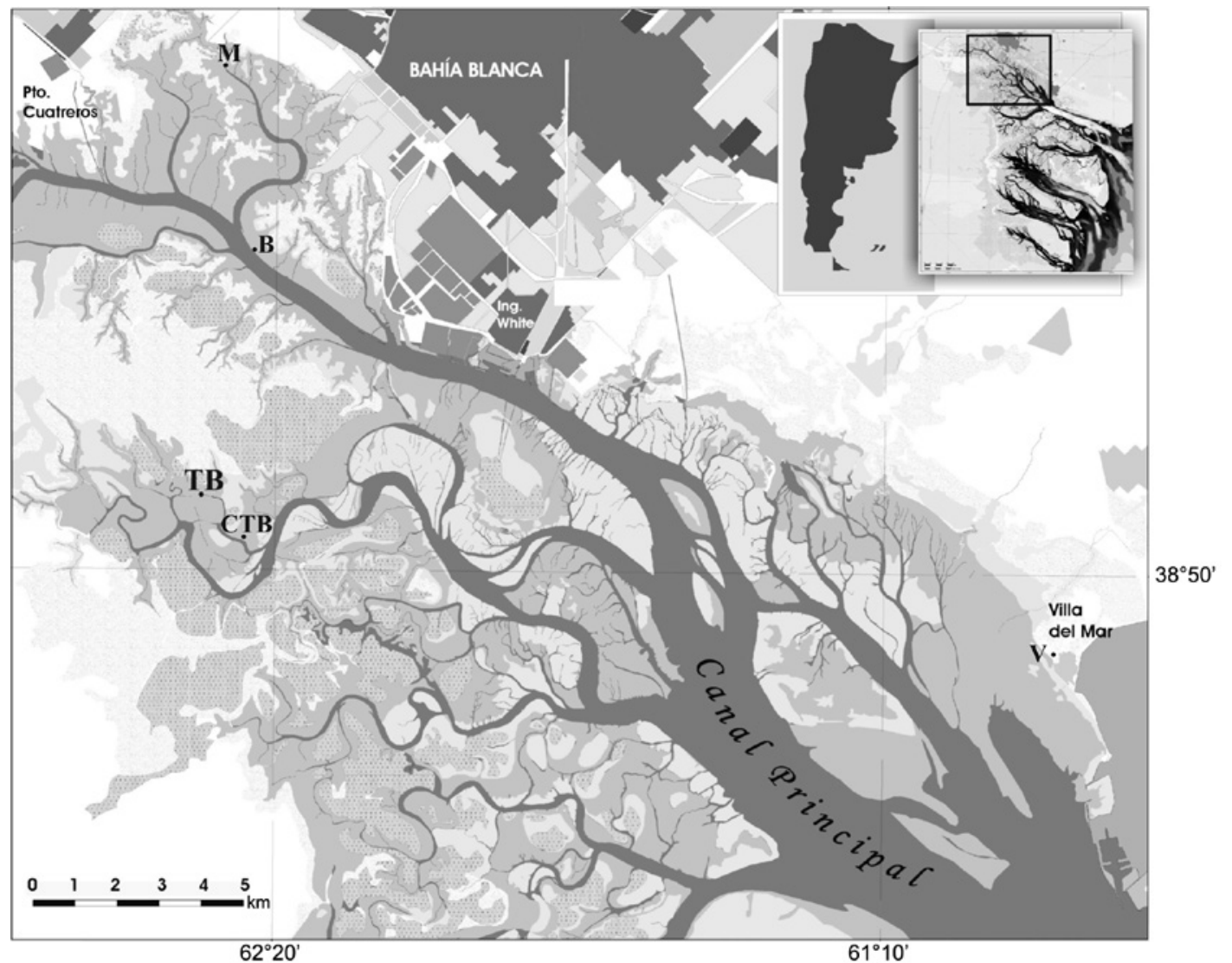

Figura 1. Mapa mostrando los sitios actuales muestreados. Abreviaturas: M, Canal Maldonado; B, Almirante Brown; V, Villa del Mar; CTB, Canal Tres Brazas y la localización del testigo TB en la zona interna del estuario de Bahía Blanca.

Figure 1. Map showing the present sites sampled. Abbreviations: M, Canal Maldonado; B, Almirante Brown; V, Villa del Mar; CTB, Canal Tres Brazas and TB core location in the inner zone of the estuary of Bahia Blanca.

Por razones de mayor simplicidad en adelante se le asignará las siglas TB a este testigo, de 4,9 m de largo. Para poder analizar los distintos proxies se lo fraccionó en 225 muestras de $2 \mathrm{~cm}$ de espesor cada una. El techo del testigo se encuentra a 1,25 $\mathrm{m}$ por encima del nivel medio del mar, siendo el rango de marea de sicigias de este lugar del orden de los 3,5 m.

\section{Metodología}

Para la extracción de las muestras actuales se utilizó un aro metálico de $10 \mathrm{~cm}$ de diámetro por $2 \mathrm{~cm}$ de alto, y se recogieron los $2 \mathrm{~cm}$ superficiales, luego el material fue desagregado con $\mathrm{H}_{2} \mathrm{O}_{2}$ de 130 volúmenes diluida en cuatro partes de $\mathrm{H}_{2} \mathrm{O}$. Posteriormente, todas las muestras fueron tamizadas en húmedo con un tamiz de 63 micrómetros de luz de malla. Una vez limpio, el residuo fue volcado en un vaso al que se le adicionó una solución de agua destilada con el colorante Rosa de Bengala ( $1 \mathrm{~g} / \mathrm{l})$. Al día siguiente, las muestras fueron lavadas a fin de eliminar el exceso de colorante y se llevaron a estufa a $50^{\circ} \mathrm{C}$. Una vez secas, se estudiaron bajo lupa binocular; se consideraron como ejemplares vivos al momento del muestreo sólo a aquellos ostrácodos que presentaron sus partes blandas teñidas de un color rosa intenso. Todos los ostrácodos presentes fueron separados mediante la técnica de picking, y ordenados en portamicrofósiles. Ambos grupos (coloreados y sin colorear) fueron utilizados para realizar estudios cualitativos y cuantitativos.

El testigo fue fraccionado en muestras cada dos centímetros, cada muestra fue desagregada con $\mathrm{H}_{2} \mathrm{O}_{2}$ de 130 volúmenes, diluida en cuatro partes de $\mathrm{H}_{2} \mathrm{O}$. Todas las muestras fueron tamizadas en húmedo con un tamiz de 63 micrómetros de luz de malla. Una vez concluido el proceso se recogió el residuo que quedó y se lo colocó en una caja de Petri. Posteriormente, las muestras fueron llevadas a estufa a $50^{\circ} \mathrm{C}$, una vez secas se procedió a recuperar mediante la técnica de picking el total de valvas de ostrácodos presentes. A fin de establecer zonaciones en la secuencia estudiada, se realizaron análisis de agrupamiento y diagramas de frecuencia. Se utilizó el programa Tilia 2.0 (Grimm, 1991), considerando sólo las especies cuya abundancia relativa es igual o superior al 5\% (Gómez et al., 2005a; Martínez, 2005). Los grupos se formaron según el método de agrupamiento jerárquico de la suma de los cuadrados del error.

Para la identificación taxonómica se siguió la clasificación genérica propuesta por Moore (1961). Para la determinación específica se utilizó bibliografía local actualizada (Bertels \& Martínez, 1990, 1997; Bertels-Psotka \& Martínez, 1999; Ferrero, 2006, 2009; Laprida, 2006; Laprida \& Ballent, 2007; Laprida \& Valero-Garcés, 2009; Whatley \& Moguilevsky, 
1975; Whatley et al., 1987, 1988; Whatley \& Cusminsky, 1995). Las fotografías fueron tomadas con microscopio electrónico de barrido Leo modelo EVO 40, del Centro Científico Tecnológico CONICET Bahía Blanca.

Boomer et al. (2003) marcan la utilidad de las asociaciones de ostrácodos para la reconstrucción de los paleoambientes sobre la base de las abundancias relativa y absoluta de las especies que la componen, las relaciones adultos/juveniles, hembras/ machos y valvas/caparazones para determinar aspectos físicos-químicos del agua, las asociaciones de vida y los procesos tafonómicos. Con el fin de evaluar las condiciones paleoambientales, en este trabajo se analizó la estructura de las poblaciones y se calculó la densidad y la frecuencia relativa de las especies. Para determinar el grado de estabilidad ambiental se calculó la diversidad faunística y el índice de diversidad de Shannon-Weaver (Shannon \& Weaver, 1949). Este índice está basado en la información contenida por un individuo en una población infinita dada por la fórmula:

$$
\mathrm{H}(\mathrm{S})=\Sigma \mathrm{Pi} . \ln \mathrm{Pi}
$$

donde $\mathrm{S}$ es el número de especies en cada muestra y Pi es la proporción para cada especie. Los valores que varían entre 2,5-3 reflejan que todas las especies están representadas equitativamente; si una especie de la asociación es muy abundante el índice de información disminuye y se relacionan con ambientes marinos inestables o restringidos (Buzas \& Gibson, 1969; Gibson \& Buzas, 1973).

Teniendo en cuenta la información proporcionada principalmente por Ornellas \& Würdig (1983), Coimbra et al. (1994, 2007), Martínez (2005), (y las referencias en él citadas) y Laprida (2006); en el presente trabajo, las especies de ostrácodos fueron agrupadas de acuerdo a los rangos de tolerancia a la salinidad en: (1) dulce- acuícolas a mixo (meso) halinas $(0-0,5 \%)$; (2) mixohalinas o salobres $(0,5-30 \%$ ) y eurihalinas o especies tolerantes a la altas variaciones en la salinidad; (3) especies euhalinas (> 30-40\%). Los rangos de salinidad se basan en el Simposio de Venecia (Hiltermann, 1985). Según dichos rangos de salinidad, en la Tabla 1 se agrupan las especies estudiadas de ambientes transicionales y marinos; con respecto a las especies no marinas o de origen continental, pueden ser dulce acuícolas o bien adaptarse a condiciones de cierta salinidad, para los fines de este trabajo se las considera en un grupo aparte sin especificar el rango de salinidad (Tabla 1).

El hallazgo de poblaciones completas, es decir, con una proporción de adultos/juveniles igual a 1:8 $(0,12)$, ocurre únicamente en ambientes con condiciones energéticas adecuadas. Si se considera la dinámica del ambiente estudiado, las tanatocenosis están sujetas a desplazamientos; sin embargo, la presencia de poblaciones con valvas adultas y determinada proporción de valvas juveniles permite establecer que, si bien pudieron haber sido removidas en algún grado, no han sido desplazadas de sus hábitats originales. Este tipo de poblaciones denominadas parautóctonas (Kidwell et al., 1986), son consideradas en este trabajo poblaciones autóctonas.

Tabla 1. Distribución de las especies estudiadas según sus rangos de salinidad. Las especies no marinas (viven en ambientes salobres o dulceacuícolas) se consideran en un grupo aparte.

Table 1. Distribution of the species according to their range of salinity. Non-marine species (living in brackish and freshwater environments) are considered in a separate group.

Cyprideis salebrosa hartmanni
Minicythere argentinensis
Leptocythere darwini
Fitales spp.
$\begin{aligned} & \text { Loxocythere variasculpta } \\ & \text { Callistocythere litoralensis } \\ & \text { Cushmanidea echevarriae } \\ & \text { Neocytherideis ruidis }\end{aligned}$

Heterocypris sp.

Limonocythere sp.

NO MARINOS

\section{RESULTADOS}

\section{Distribución cualitativa y cuantitativa de los ostrácodos actuales}

En todas las transectas la salinidad aumenta con la elevación topográfica; alcanzando los valores más altos en los salt pans (cubetas salinas) que aparecen en elevaciones cercanas a las de las pleamares extraordinarias, donde la evaporación es máxima. Sin embargo, en los sitios elevados, pero con cobertura de Sarcocornia perennis la salinidad es notablemente menor que en los salts pans vecinos, sugiriendo que esta especie arbustiva tendría un efecto moderador de la salinidad (Tabla 2).

En las marismas altas con Sarcocornia perennis y que presentan salinidades que varían entre $24-32,8 \%$, no se hallaron ostrácodos. En las cubetas salinas o salt pan del Canal Maldonado y Villa del Mar con salinidades variables que no superan los 34,8\% se registra la asociación de Cyprideis salebrosa hartmanni y Callistocythere litoralensis con poblaciones poco desarrolladas. La cubeta salina de Almirante Brown resultó estéril con una salinidad de 46,4 \%o.

En las marismas de los distintos sitios estudiados se reconocen diferentes asociaciones de ostrácodos: (i) en las marismas bajas con Spartina alterniflora se hallan dos asociaciones: a) la asociación de Neocytherideis ruidis y Loxocythere variasculpta, registrada en la desembocadura del Canal Maldonado en la porción interior; b) la asociación de Cyprideis salebrosa y Callistocytere litoralensis con poblaciones poco desarrolladas, hallada en la porción intermedia del estuario (Villa del Mar) donde se encontraron muy pocos ejemplares; (ii) finalmente, en la marisma de S. alterniflora del Canal Tres Brazas se halla la asociación de C. litoralensis dónde esta especie es dominante. 
Tabla 2. Variables ambientales actuales en las estaciones de muestreo. Abreviaturas: B, Almirante Brown; V, Villa del mar; M, Canal Maldonado; CTB, Canal Tres Brazas.

Table 2. Modern environmental variables at sampling stations. Abbreviations: B, Almirante Brown; V, Villa del mar; M, Canal Maldonado; CTB, Canal Tres Brazas.

\begin{tabular}{clcc}
\hline Muestras & Tipo de ambiente & Salinidad (ups) & PH \\
\hline B1 & Marisma alta cerca de S. perennis & 24,3 & 7,96 \\
B2 & Cuenca salina (Salt pan) & 46,4 & 8,24 \\
B3 & Llanura de marea & 30,6 & 7,88 \\
V1 & Marisma baja con S. alterniflora & 18,8 & 7,96 \\
V2 & Marisma baja con S. alterniflora & 10,6 & 7,86 \\
V3 & Cuenca salina (Salt pan) & 34 & 8,24 \\
V4 & Marisma alta con S. perennis & 32,8 & 8,35 \\
M1 & Marisma baja & 23 & 7,88 \\
M2 & Marisma baja con S. alterniflora & 21,4 & 7,95 \\
M3 & Marisma baja con S. alterniflora & 22,8 & 8,2 \\
M4 & Marisma baja con S. densiflora & 17,6 & 7,89 \\
M5 & Marisma alta con S. perennis & 31 & 7,92 \\
M6 & Cuenca salina (Salt pan) & 34,8 & 8,28 \\
M7 & Planicie de marea & 16,9 & 7,96 \\
CTB1 & Planicie de marea & 34,8 & 7,90 \\
CBT2 & Marisma con Spartina & 35 & 8,00 \\
CTB3 & Planicie de marea & 33,7 & 7,70 \\
CTB4 & Marisma con Spartina & 34 & 8,20 \\
CTB5 & Marisma con Spartina & 32 & 7,90 \\
CTB6 & Planicie de marea & 35 & 7,80 \\
\hline
\end{tabular}

En la planicie de marea del Canal Maldonado se halló la asociación de Cyprideis salebrosa hartmanni y Callistocythere litoralensis con poblaciones muy desarrolladas; por otro lado la llanura de marea de Almirante Brown con una salinidad de $30,6 \%$ resultó estéril (Tabla 3).

\section{Relación entre las variaciones ambientales y la fauna de ostrácodos}

En las cubetas salinas la elevada salinidad y la desecación por exposición aérea, condiciona el desarrollo de las poblaciones de ostrácodos. Las cubetas son cuerpos de agua de poca profundidad formados en depresiones de los sedimentos intermareales; Cyprideis salebrosa hartmanni es la especie dominante en este tipo de ambiente, ya que su amplio nicho ecológico le permite desarrollarse en condiciones de salinidad elevada e inestable. La diferencia en la salinidad de los sitios muestreados puede deberse a que en el Canal Maldonado y Villa del Mar las cubetas salinas se encuentran rodeadas por matas de Sarcocornia perennis que en gran medida las protegen de la desecación y, consecuentemente, producen un efecto moderador en la salinidad que permiten el desarrollo de C. salebrosa hartmanni. En cambio, en Almirante Brown por ausencia de vegetación las cubetas salinas se encuentran expuestas y por ello presentan mayor tasa de evaporación y mayor concentración de sales, condiciones ambientales más de desfavorables para el desarrollo de los ostrácodos.

Las marismas se caracterizan por estar sujetas a un largo período de emersión y consecuente evaporación, lo que provocan el ascenso capilar de los elementos nutritivos hacia la película superficial. Este ascenso de nutrientes es favorecido por la estructura heterogénea del sedimento fino, con una proporción adecuada de arena. La vegetación, por su parte, impide la desecación, retiene las partículas alimenticias transportadas por las corrientes y aporta oxígeno necesario para la degradación de la materia orgánica (Carbonel, 1980). Todas las especies registradas en las marismas estudiadas son mixohalinas y eurihalinas adaptadas a condiciones ambientales inestables. Probablemente, las diferentes asociaciones registradas en las marismas actuales se relacionan principalmente con las variaciones de salinidad, tróficas e hidrodinámicas del medio de los distintos sitios. La escasez de ostrácodos recuperados de las muestras de las marismas de Villa del Mar se debe a las condiciones hidrodinámicas propias de un ambiente transicional entre los ambientes dominados por sedimentos finos y las costas arenosas. En las marismas altas con Sarcocornia perennis el factor condicionante en el desarrollo de la ostracofauna puede ser la desecación debida a la frecuente exposición aérea. Estos ambientes, emergidos durante prolongados períodos de tiempo durante el día, son desfavorables para la nutrición de los ostrácodos bentónicos, los cuales dependen de los nutrientes que se hallan en la película superficial del agua en contacto con los primeros milímetros de sedimento (Carbonel, 1980).

La ausencia de ostrácodos en las planicies de marea de Almirante Brown puede atribuirse a las altas densidades de cangrejos Chasmagnathus granulatus Dana y el efecto 
Tabla 3. Número de individuos total (T) y vivos (V) por muestra de cada especie autóctona en 25 grs. de sedimento. Abreviaturas: $\mathbf{B}$, Almirante Brown; V, Villa del mar; M, Canal Maldonado; CTB, Canal Tres Brazas.

Table 3. Total number of individuals ( $T$ ) and live (V) per sample of each autochthonous species in $25 \mathrm{~g}$. of sediment. Abbreviations: B, Almirante Brown; V, Villa del mar; M, Canal Maldonado; CTB, Canal Tres Brazas.

\begin{tabular}{|c|c|c|c|c|c|c|c|c|c|c|c|c|}
\hline \multirow{2}{*}{ istras } & \multicolumn{2}{|c|}{ C.s.hartmanni } & \multicolumn{2}{|c|}{ C.multidentata } & \multicolumn{2}{|c|}{ L.darwini } & \multicolumn{2}{|c|}{ N.ruidis } & \multicolumn{2}{|c|}{ C.litoralensis } & \multicolumn{2}{|c|}{ L.variasculpto } \\
\hline & $\mathrm{T}$ & $\mathrm{V}$ & $\mathrm{T}$ & V & $\mathrm{T}$ & V & $\mathrm{T}$ & V & $\mathrm{T}$ & V & $\mathrm{T}$ & V \\
\hline M1 & 7 & 6 & 1 & 1 & 0 & 0 & 7 & 6 & 6 & 6 & 3 & 3 \\
\hline M2 & 10 & 8 & 1 & 1 & 0 & 0 & 6 & 6 & 5 & 5 & 5 & 5 \\
\hline M3 & 2 & 2 & 0 & 0 & 0 & 0 & 0 & 0 & 0 & 0 & 30 & 26 \\
\hline M4 & 0 & 0 & 2 & 2 & 2 & 2 & 0 & 0 & 0 & 0 & 40 & 38 \\
\hline M5 & 40 & 20 & 0 & 0 & 0 & 0 & 0 & 0 & 0 & 0 & 0 & 0 \\
\hline M6 & 4 & 0 & 0 & 0 & 0 & 0 & 0 & 0 & 0 & 0 & 0 & 0 \\
\hline M7 & 116 & 110 & 0 & 0 & 0 & 0 & 0 & 0 & 0 & 0 & 68 & 48 \\
\hline B4 & 53 & 46 & 0 & 0 & 0 & 0 & 0 & 0 & 0 & 0 & 0 & 0 \\
\hline V1 & 27 & 8 & 0 & 0 & 0 & 0 & 0 & 0 & 0 & 0 & 6 & 6 \\
\hline V2 & 24 & 5 & 0 & 0 & 0 & 0 & 0 & 0 & 0 & 0 & 5 & 5 \\
\hline V3 & 23 & 13 & 0 & 0 & 0 & 0 & 0 & 0 & 0 & 0 & 4 & 4 \\
\hline V4 & 16 & 0 & 0 & 0 & 0 & 0 & 0 & 0 & 0 & 0 & 0 & 0 \\
\hline СТB1 & 8 & 8 & 1 & 1 & 0 & 0 & 9 & 9 & 6 & 6 & 0 & 0 \\
\hline СТВ2 & 5 & 5 & 2 & 2 & 2 & 2 & 0 & 0 & 5 & 5 & 38 & 38 \\
\hline СТВ3 & 4 & 4 & 0 & 0 & 5 & 5 & 9 & 9 & 0 & 0 & 0 & 0 \\
\hline CTB4 & 0 & 0 & 0 & 0 & 0 & 0 & 10 & 10 & 0 & 0 & 2 & 2 \\
\hline CTB5 & 10 & 10 & 0 & 0 & 2 & 2 & 12 & 12 & 0 & 0 & 9 & 9 \\
\hline CTB6 & 37 & 37 & 0 & 0 & 18 & 18 & 62 & 62 & 1 & 1 & 0 & 0 \\
\hline
\end{tabular}

bioturbador de los mismos. Los cangrejos en tan altas densidades son agentes modeladores del paisaje por la gran cantidad de sedimentos que movilizan, además de su comportamiento como herbívoros y predadores de otros organismos bentónicos (Calvo Marcilese \& Pratolongo, 2009).

\section{Sedimentos del Holoceno}

El testigo se caracteriza por presentar en su base (490 a $250 \mathrm{~cm}$ ) una arena fina limosa de color grisáceo con capas laminares y lenticulares de limo predominantes principalmente en la base; la zona media $(250$ a $135 \mathrm{~cm})$ está formada por depósitos de arena media con intercalaciones de arena fina limosa, sin ningún tipo de estructura visible pero con fragmentos de conchilla. El tramo superior (135 a $0 \mathrm{~cm}$ ) del testigo está compuesto por arena fina limosa con intercalaciones de limo, terminando hacia el techo con un bloque homogéneo de limo de coloración rojiza con restos orgánicos (Figura 2).

\section{Dataciones radiocarbónicas}

En el testigo se realizaron cuatro fechados radiocarbónicos donde las edades radiocarbónicas convencionales (conv.) fueron calibradas a edades siderales o calibradas (cal.) empleando el método de la curva de calibración Calib. 7.0 (http://calib.qub.ac.uk/calib/). Debido al ambiente depositacional que se determina más adelante para la calibración se empleó el conjunto de datos atmosféricos para el hemisferio sur, SHcal13 a $2 \sigma$ (Hogg et al., 2013), en lugar del conjunto de la curva marina Marine04 (Hughen et al.,
2004). Ello obedeció a que el carbono (C) que se incorpora en el ambiente intermareal del estuario de Bahía Blanca sería primariamente de origen atmosférico (Gómez et al., 2008).

Este testigo cuenta con cuatro dataciones radiocarbónicas, las cuales fueron realizadas sobre fango orgánico. La primera de ellas, se halla a los 488,89 cm de profundidad y arrojó una edad conv. de 5.090 \pm 40 AP (BETA 282197) (cal. 5.662-5.907 años AP), la segunda a los $280 \mathrm{~cm}$ con una edad convencional

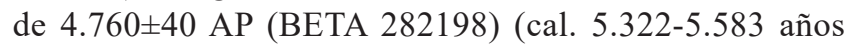
AP), la tercera ubicada a los $231,11 \mathrm{~cm}$ de profundidad arrojó una edad convencional de 4.040 \pm 70 AP (BETA KIA 42949) (cal. 4.240-4.808 años AP), mientras que la cuarta datación obtenida a los $71,11 \mathrm{~cm}$ de profundidad dio una edad convencional de 3.188 \pm 64 AP (ARIZONA x28022) (cal. 3.176-3.553 años AP).

\section{SISTEMÁTICA}

Subclase OSTRACODA Latreille, 1806

Orden PODOCOPIDA Müller, 1894

Suborden PODOCOPINA Sars, 1865

Superfamilia CYTHERACEA Baird, 1850

Familia CYTHERIDAE Baird, 1850

Heterocypris Claus, 1893

Heterocypris sp.

(Figura 3A) 


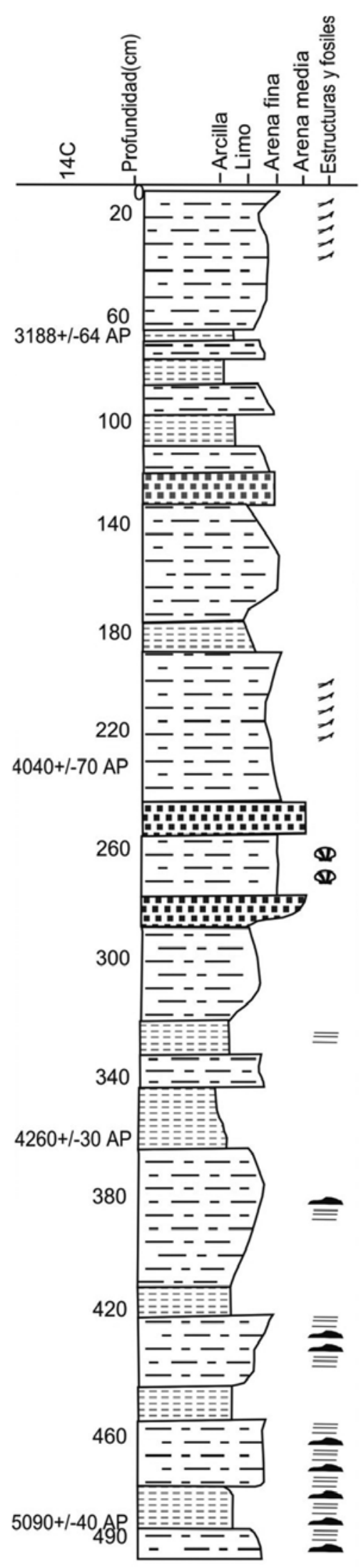

[1.5A Arena

E:- Arena fina limosa $-\exists$ Limo QDestos de conchillas $\sim$ Materia orgánica Capa lenticular Capa laminar
Material fotografiado. MiUNS 308, VI, largo: $0,16 \mathrm{~mm}$; alto: $0,63 \mathrm{~mm}$ (M 325).

\section{Loxocythere Hornibrook, 1952}

Loxocythere variasculpta Whatley, Moguilevsky, Toy, Chadwick \& Ramos, 1997

(Figura 3B)

1990 Perissocytheridea sp. n.; Bertels \& Martínez, lám. 4, fig. 39. 1995 Loxocythere sp.; Whatley et al., p. 22, lám. 1, figs.13,14. 1997 Loxocythere variasculpta sp. n.; Whatley et al., p. 158159, lám. 2, figs.13,16.

1997 Perissocytheridea whitensis sp. n.; Bertels \& Martínez, p. 330-331, lám. 2, figs. 1-6, lám. 6, fig. 2.

1998 Loxocythere variasculpta sp. n.; Whatley et al., pags. 158-159, lám. 2, figs. 13-16.

2006 Perissocytheridea sp.; Laprida, p. 189, 196-197, figs. 3.F,6.E-F.

2009 Loxocythere variasculpta Whatley, Moguilevsky, Toy, Chadwick \& Ramos, 1997. Ferrero, p. 654.

Material fotografiado. MiUNS 309 (1), Caparazón, largo: 0,41 mm; alto: 0,19 mm; ancho. 0,03 mm (M. 11), MiUNS 309 (2), VI, largo: 0,40 mm; alto: 0,20 mm (M. 301).

Familia CYTHERIDEIDAE Sars, 1925 Subfamilia CYTHERIDEINAE Sars, 1925

Cyprideis Jones, 1857

Cyprideis salebrosa hartmanni Ramírez, 1967

(Figuras 3C-D)

1967 Cyprideis hartmanni n. sp. Ramírez, p. 40-42, lám. 11, figs. 74-79; lám. 12, figs. 80-89.

1983 Cyprideis salebrosa hartmanni Ramírez, 1967, emend. Ornellas \& Würdig, subsp. nov. Ornellas \& Würdig, p. $97-$ 102, lám. I, figs. 1-8.

1986 Cyprideis salebrosa van den Bold, 1963; Zabert, p. 218, lám. II, figs. 5a-e; lám. III, figs. 14a-b.

1988 Cyprideis salebrosa van den Bold 1963; Dias-Brito, Moura \& Würdig, lám. 1, fig. 14.

1990 Cyprideis salebrosa hartmanni Ramírez, 1967; Bertels \& Martínez, lám. 1, fig. 7.

1996 Cyprideis salebrosa hartmanni Ramírez, 1967; Ferrero, p. 218. lám. 2, figs. 1a-b.

1997 Cyprideis salebrosa hartmanni Ramírez, 1997; Bertels \& Martínez, p. 329-330, lám. 1, figs. 10-11.

1998 Cyprideis salebrosa van den Bold, 1963; Whatley et al., p. 94, lám. 1, figs. 24, 25.

2005 Cyprideis salebrosa hartmanni Ramírez, 1967; Martínez, p. 672.

2006 Cyprideis salebrosa Van den Bold, 1963; Laprida, p.189, 194, 197; figs. 3D-E; 6B-D.

2009 Cyprideis salebrosa hartmanni Ramírez, 1967; Ferrero, p. 654-655, fig. 9.5 .

Figure 2. TB core lithological profile. 
Material fotografiado. MiUNS 310 (1), VD masculina, largo: 0,98 mm; alto: 0,55 mm (M 309); MiUNS 310 (2), VD feminina, largo: 0,96 mm; alto: 0,54 mm (M 309).

Neocytherideis Puri, 1952

Neocytherideis ruidis Whatley, Moguilevsky, Chadwick, Toy y Ramos 1998

(Figura 3E)

1990 Neocytherideis sp. Bertels \& Martínez, lám. 2, fig. 18. 1997 Neocytherideis sp. Whatley et al., p.164, lám.3, fig. 16. 1997 Neocyterideis sp. n. Bertels \& Martínez, p. 333, lám. 2, figs. 11-12, lam. 6, fig. 6 .

1998 Neocytherideis ruidis sp. n. Whatley et al., p. 96,98, lám. 2, figs. 12-16.

2005 Neocytherideis ruidis Whatley et al., 1998; Martínez, p. 672. 2009 Neocytherideis ruidis Whatley, Moguilevsky, Chadwick, Toy \& Ramos 1998; Ferrero, p. 654.

Material fotografiado. MiUNS 311, VI, largo: 0,45 mm; alto: 0,18 mm (M. 6).

Cushmanidea Blake, 1933

Cushmanidea echevarriae Bertels \& Martínez, 1997

(Figura 3G)

1987 Cushmanidea sp. Echevarría, p. 134, lám. II, fig. j. 1988 Cushmanidea sp. Echevarría, p. 324, lám. I, fig. d. 1990 Cushmanidea sp. n. Bertels \& Martínez, lám. 2, fig. 20. 1997 Cushmanidea? echevarriae sp. nov. Bertels \& Martínez, p. 334-335, lám.2, figs. 15-17, lám.6, fig.8.

1998 Cushmanidea variopunctata sp. nov. Whatley et al., p. 98, lám.2, figs. 17-20.

2005 Cushmanidea ?echevarriae Bertels \& Martínez, 1997; Martínez, p. 672.

2009 Cushmanidea echevarriae Bertels \& Martínez, 1997; Ferrero, p. 654.

Material fotografiado. MiUNS 312 (1), VD, largo: 0,55 mm; alto: 0,21 mm (M. 301); MiUNS 312 (2), VD. Largo. 0,54 $\mathrm{mm}$; alto. 0,22 mm (M. 301).

Minicythere Ornellas, 1974

Minicythere argentinensis Bertels \& Martínez, 1997 (Figura 3F)

1975 Cushmanidea n. sp.; Bertels, lám. 5, fig. 13. 1978 Minicythre sp.; Zabert, p. 135-136, lám. VI, fig. 41. 1990 Cushmanidea sp. 2; Bertels \& Martínez, lám. 3, fig. 21. 1997 Minicythere argentinensis Bertels \& Martínez, p. 336337, lám. 2, figs.18-21, lám. 6, fig. 9.

2005 Minicythere argentinensis Bertels \& Martínez, 1997; Martínez, p. 672.

2009 Minicythere argentinensis Bertels \& Martínez, 1997; Ferrero, p. 654.
Material fotografiado. MiUNS 313, VI, largo: 0,47 mm; alto: 0,19 mm (TB testigo; M.305).

Familia LEPTOCYTHERIDAE Hanai, 1957

Leptocythere Sars, 1924

Leptocythere darwini Whatley et al., 1997

(Figuras 3I-J)

1975 Leptocythere n. sp. A. Whatley \& Moguilevsky, p. 511512. lám. I, figs. 4-6; lám. III, figs., 1- 7.

1975 Leptocythere n. sp. B. Whatley \& Moguilevsky, p. 512513, lám. I, figs. 7-11; lám. III, figs. 8-15, 20.

1990 Leptocythere sp. n. Bertels \& Martínez, lám. 4, fig. 31. 1997 Leptocythere darwini sp. nov. Whatley, Moguilevsky, Toy, Chadwick \& Ramos, p. 185-186, lám. 7, figs. 7, 9.

1997 Leptocythere sp. n.. Bertels \& Martínez, p. 48-49, lám. 4, figs. 8-12, lám. 6, fig. 13 .

Material fotografiado. MiUNS 314 (1), VI, largo: 0,36 mm; alto: 0,17 mm (M. 279); MiUNS 314 (2) VD, largo: 0,39 mm; alto: $0,15 \mathrm{~mm}$ (M. 301).

\section{Callistocythere Ruggieri, 1953}

Callistocythere litoralensis (Rossi de García), 1966

(Figura 3H)

1966 Perissocytheridea litoralensis sp. n. Rossi de García, p. 206, lám. II, figs. 3a-c.

1975 Callistocythere n. sp. Bertels, lám. 5, fig. 3.

1975 Callistocythere litoralensis (Rossi de García); Whatley \& Moguilevsky, lám. II, figs. 10-11.

1975 Callistocythere sp. A. Whatley \& Moguilevsky, p. 515-516, lám. I, figs. 16-18; lám. II, figs. 1-3; lám. III, figs. 16-19, 22.

1977 Callistocythere litoralensis (Rossi de García) 1966; Zabert \& Herst, p. 154, lám. II, fig. 8.

1978 Callistocythere litoralensis (Rossi de García) 1966; Zabert, p. 133-135, lám. VI, figs. 39a-b.

1979 Callistocythere litoralensis (Rossi de García); Sanguinetti, p. 61, lám. 4, figs. 1c, 2a.

1982 Callistocythere sp. Bertels, Kotzian \& Madeira-Falcetta, p. 148-149, lám VII, figs. 3a-b.

1988 Callistocythere sp. 2. Dias-Brito, Moura \& Würdig, lám. 1, fig. 12.

1988b Callistocythere litoralensis Rossi de García, 1969; Echevarría, p. 334, lám. III, fig. c.

1990 Callistocythere litoralensis (Rossi de García, 1966); Bertels \& Martínez, lám. 4, fig. 32.

1997 Callistocythere nucleoperiscum sp nov. Whatley, Moguilevsky, Toy, Chadwick \& Ramos, p. 184-185, lám.7, figs. 5, 6 .

1997 Callistocythere litoralensis (Rossi de García, 1966); Bertels \& Martínez, p. 49, lám. 4, figs. 13-14.

1998 Callistocythere litoralensis (Rossi de García. 1966); 
Bertels-Postka \& Laprida, p. 118-119, lám. 2, figs. 14-15. 1998 Callistocythere nucleoperiscum Whatley et al.; Whatley et al., p. 104, lám. 4, figs. 7-8.

2005 Callistocythere litoralensis (Rossi de García, 1966); Martínez, p. 672.

2009 Callistocythere litoralensis (Rossi de García. 1966); Ferrero, p. 656, fig. 10.2.

Material fotografiado. MiUNS 315 (1), VI, largo: 0,38 mm; alto: 0,20 mm (M. 301).

Familia LIMNOCYTHERIDAE Klie, 1938

Limnocythere Brady, 1868

Limnocythere sp.

(Figura 4A)

Material fotografiado. MiUNS 316 (1), VD, largo: 0,57 mm; alto: 0,27 mm (M. 32) macho, MiUNS 316 (2), VD, largo: $0,46 \mathrm{~mm}$; alto: $0,24 \mathrm{~mm}$ (M. 32) hembra.

Observación. Si bien es asemejable a Limnocythere cusminskyae Mercau et al., 2014 debido al escaso material recuperado y a que la mala preservación del mismo no permite observar en detalle las características taxonómicas y la ornamentación de la valva se ha decidido dejar la asignación abierta.

\author{
Familia PARADOXOSTOMATIDAE \\ Brady \& Norman, 1889 \\ Subfamilia CYTHEROMATINAE Elofon, 1939
}

Pellucistoma Coryell \& Fields, 1937

Pellucistoma elongata Whatley, Moguilevsky, Chadwick, Toy \& Feijó Ramos, 1998

(Figuras 4B-C)

1975 Pellucistoma n. sp. Bertels, lám. 5, fig. 14.

1990 Pellucistoma sp. n. Bertels \& Martínez, lám. 4, fig. 37. 1997 Pellucistoma sp. Bertels \& Martínez, p. 52-53, lám. 6, fig. 21.

1997 Pellucistoma elongata sp. n. Whatley et al., p. 53, lám. 8, figs. 14-18.

1998 Pellucistoma elongata sp.n. Whatley et al.; Whatley et al., p. 106, 108, lám. 4, figs. 15-16 m.

2005 Pellucistoma elongata Whatley et al., 1998; Martínez, p. 672. 2009 Pellucistoma elongata Whatley et al.; Ferrero, p. 656.

Material fotografiado. MiUNS 318, (1) VD, largo: 0,50 mm; alto: 0,26 mm (M.105), MiUNS 318, (2) caparazón VD, largo: $0,53 \mathrm{~mm}$; alto: $0,29 \mathrm{~mm}$ (M.105).

Cytherois sp.

(Figura 4D)

1997 Cytherois sp. 4; Whatley et al., p. 59, lám. 9, figs. 4-6.
Material fotografiado. MiUNS 319, VD, largo: 0,48mm; alto: $0,21 \mathrm{~mm}(\mathrm{M} 2)$.

Descripción. Caparazón alargado reniforme, superficie de la valva lisa; margen anterior y posterior ahusados. Máxima altura en la mitad de la valva. Charnela adonta, vestíbulos poco desarrollados o ausentes.

Familia XESTOLEBERIDIDAE Sars, 1928

Xestoleberis Sars, 1928

Xestoleberis sp.

(Figura 4E)

1997 Xestoleberis sp. 1; Whatley et al., p. 210, lám. 13, fig. 11, 12.

Material fotografiado. MiUNS: 320 , VD, largo: 0,33mm; alto: $0,16 \mathrm{~mm}$ (M. 5).

Descripción. Vista externa. Valvas subovoides lisas con los extremos anterior y posterior estrechos. Borde dorsal convexo en la zona media y levemente oblicuo hacia los extremos. Borde ventral recto. Borde anterior y posterior redondeados, no hay diferencia muy marcada entre ambos bordes. Altura máxima hacia la mitad de la altura valvar. No fue posible observar las características internas de la valva debido a que todos los ejemplares recuperados estaban rellenos con sedimento.

Semixestoleberis Hartmann, 1962

Semixestoleberis sp.

(Figura 4F)

Material fotografiado. MiUNS: $321, \mathrm{VD}$, largo: $0,376 \mathrm{~mm}$; alto: 0,211 mm (M. 17).

Descripción. Vista externa VD. En vista lateral, contorno subovalado, borde dorsal convexo, borde ventral subrecto, con suave concavidad hacia la región anterior, borde anterior muy oblicuamente redondeado y posterior redondeado. La VI se diferencia de la VD por poseer un borde anterior más alto y un borde posterior oblicuamente redondeado. Mayor altura en la mitad posterior. Valvas lisas. No se observó dimorfismo sexual. Vista interna: VD. Lamela interna desarrollada con vestíbulo anterior y posterior, siendo el anterior más amplio. Zona marginal angosta, con numerosos canales poros marginales simples. Se distinguen tres impresiones del músculo aductor, algo cóncavas hacia el margen anterior y una impresión mandibular redondada. Chanela lofodonta con el diente anterior más desarrollado y un surco liso.

\section{Semixestoleberis $\mathrm{sp} .1$}

(Figura 4G)

Descripción. Valvas muy pequeñas y, en vista lateral, ovoides. Vista externa VI. Borde dorsal regularmente convexo y ventral algo sinuoso en la mitad de la longitud; borde anterior apenas oblicuamente redondeado y posterior 
ampliamente redondeado. Mayor altura hacia la mitad del caparazón. La VD se distingue de la VI por presentar el borde anterior oblicuamente redondeado y el borde ventral con una sinuosidad en la mitad anterior del caparazón. No se observó dimorfismo sexual. Vista interna. Lamela y vestíbulo anteriores más desarrollados. Zona marginal angosta, con numerosos poros de canales rectos y simples. Poros de canales normales cribosos. Cuatro impresiones del músculo aductor dispuestos en hilera subvertical; impresión frontal grande y en forma de V; dos impresiones mandibulares alargadas. Charnela adonta. Material fotografiado. MiUNS: 322, VI, largo: 0,320 mm; alto: $0,210 \mathrm{~mm}$ (M. 8).

\section{Semixestoleberis debueni Hartmann, 1962}

(Figura 4H)

1962 Semixestoleberis debueni sp. n. Hartmann, p. 231-233, figs. 160-172.

1990 Semixestoleberis debueni Hartmann, 1962; Bertels \& Martínez, lám. 4, fig. 42.

1997 Semixestoleberis debueni Hartmann, 1962; Whatley et al., p. 211, lám. 12, fig.7,9.

1997 Semixestoleberis debueni Hartmann, 1962. Bertels \& Martínez, p. 348, 352, 354, 360, lám. 5, figs. 14-18, lám.6, fig. 22.

2005 Semixestoleberis debueni Hartmann, 1962; Martínez, p. 672 .

Material fotografiado. MiUNS: 323, VD, largo: 0,35 mm; alto: $0,18 \mathrm{~mm}$ (M287).

\section{Distribución de los ostrácodos del Holoceno}

Se registraron un total de 11 géneros representados por 13 especies de ostrácodos bentónicos, cuyas valvas presentan buen estado de preservación. En cuanto a la fauna acompañante, sólo se hallan ejemplares de Littoridina australis (d'Orbigny).

A lo largo del testigo, los valores de densidad fluctúan entre 597 y 15 individuos y los índices de Shanonn-Weaver son bajos, variando entre 1,3 y 0,13 (Figura 5). La información obtenida de los análisis cualitativos y cuantitativos realizados sobre las muestras de ostrácodos recuperados del testigo permitió definir tres zonas con la utilización del programa Thilia 2.0 (Grimm, 1991) (Figura 6).

Zona I $(498,89-322,23 \mathrm{~cm})$. Dominan las especies mixoeuhalinas Neocytherideis ruidis, Loxocythere variasculpta y Callistocythere litoralensis (Figura 6), esta última especie es muy abundante y en algunos casos dominante en muestras actuales de sedimentos del Canal Tres Brazas. Asociados a estos ostrácodos se hallan poblaciones de especies mixohalinas y eurihalinas Cyprideis salebrosa hartmanni y Leptocythere darwini. Los ostrácodos fitales y parafitales (Pellucystoma elongata, Cytherois sp., Xestoleberis sp. y Semixestoleberis sp.), si bien no son muy abundantes, sus poblaciones presentan ejemplares adultos y juveniles; es probable que los valores cuantitativos de estas especies se relacionen con la fragilidad de sus valvas.
Zona II (322,23-166,67cm). Casi todas las muestras resultaron estériles y fueron abundantes los caparazones de gastrópodos de Littoridina australis (d'Orbigny).

Zona III $(166,67-000 \mathrm{~cm})$. Se destaca la dominancia de Loxocythere variasculpta, Neocytherideis ruidis y Cyprideis salebrosa hartmanni. Callistocythere litoralensis es abundante y presenta numerosos caparazones. Asociadas a estas especies se hallan los ostrácodos fitales Pellucystoma elongata y Cytherois sp.. En este sector del testigo se registran las especies de origen continental Limnocythere sp. y Heterocypris sp.. Finalmente, a partir de los $80 \mathrm{~cm}$ no se registran ostrácodos.

\section{DISCUSIÓN}

\section{Interpretaciones paleoambientales}

A lo largo del testigo se registran los valores del índice de Shanonn-Weaver inferiores a 2,5 (Figura 5), situación que se relaciona con la baja diversidad de especies autóctonas (con un valor máximo de 10 especies) y con el desarrollo de las especies más abundantes. Asimismo las oscilaciones de los valores de densidad (Figura 5) indican condiciones ambientales de salinidad, sustrato y vegetación inestables (Cronin, 1979).

En la base del testigo $(498,89-322,22 \mathrm{~cm})$ la dominancia de especies mixo-euhalinas y la abundancia de las especies mixohalinas permiten determinar que los sedimentos fueron depositados en un ambiente con fluctuaciones en la salinidad. Por otra parte, la presencia de ostrácodos relacionados con la vegetación como Pellucystoma elongata, Cytherois sp., Xestoleberis sp. y Semixestoleberis sp. reflejan el desarrollo de marismas. Todas las especies mencionadas en este sector del testigo han sido encontradas en sedimentos actuales de marismas y planicies de marea del estuario de Bahía Blanca; entre ellas, Neocytherideis ruidis y Leptocythere darwini son exclusivas de dichos ambientes (Martínez, 2005; Kihn, 2015) y por consiguiente, la dominancia de especies mixo-euhalinas, reflejan condiciones ambientales de salinidad inestable. Por otra parte, el desarrollo de poblaciones completas y altas densidades de ostrácodos indican condiciones tróficas favorable para su desarrollo, lo que sumado a la diversidad de taxa relacionada con la vegetación, indican que los sedimentos de la base del testigo se originaron en un ambiente intermareal con marismas desarrolladas.

El sector medio (322,22-166,67 cm), la marcada disminución de la ostracofauna, la presencia de fragmentos de conchilla y los caparazones de gastrópodos pertenecientes a la especie Littoridina australis (d'Orbigny) que han sido transportados indicarían un aumento notable de la energía ambiental, lo que podría obedecer a una profundización del ambiente.

En el tramo superior $(166,67-00 \mathrm{~cm})$ el aumento en la densidad y diversidad de la ostracofauna con respecto a los niveles medios y la dominancia de especies mixoeuhalinas sumado a la presencia de especies fitales y de aguas continentales permiten establecer un ambiente de marisma con marcada influencia continental. 


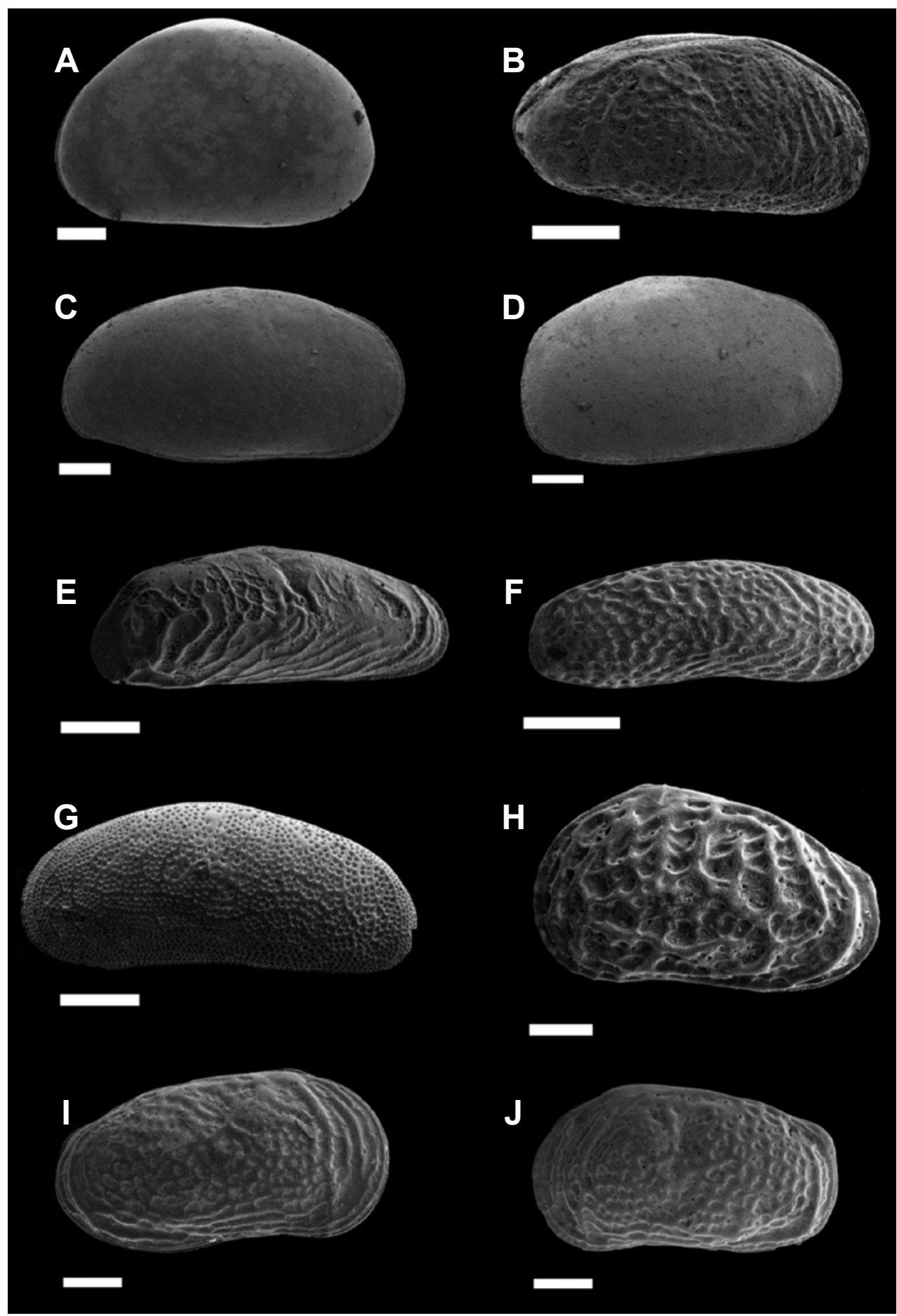

Figura 3. A, Heterocypris sp. (x244), vista externa VI (MiUNS 308, M 325). B, Loxocythere variasculpta Whatley et al., 1997 (x706), vista lateral derecha (MiUNS 309 (1), M 11). C-D, Cyprideis salebrosa hartmanni Ramírez, 1967, C, (x329), vista externa VD macho (MiUNS 308 (1), M 339); D, (x390), vista externa VI hembra (MiUNS 308 (2), M 339). E, Neocytherideis ruidis Whatley et al., 1998 (x660), vista externa VD (MiUNS 310, M 6). F, Minicythere argentinensis Bertels \& Martínez, 1997 (x649), vista externa VI (MiUNS 313, M 305). G, Cushmanidea echevarriae Bertels \& Martínez, 1997 (x588), vista externa VD (MiUNS 312, M 301). H, Callistocythere litoralensis (Rossi de García) 1966 (x868), vista externa VI (MiUNS 329 (1), M 301). I, Leptocythere darwini Whatley et al., 1997 (x682), vista externa VI hembra (MiUNS (1), M 279); J, Leptocythere darwini Whatley et al., 1997 (x582), vista externa VD macho (MiUNS 328(2), M 301). Escalas $=30 \mu \mathrm{m}$.

Figure 3. A, Heterocypris sp. (x244), external view LV (MiUNS 308, M 325). B, Loxocythere variasculpta Whatley et al., 1997 (x706), lateral view C (MiUNS 309 (1), M 11). C-D, Cyprideis salebrosa hartmanni Ramírez, 1967, C, (x329), external view male RV (MiUNS 308 (1), M 339); D, (x390), external view female LV (MiUNS 308 (2), M 339). E, Neocytherideis ruidis Whatley et al., 1998 (x660), external view RV (MiUNS 310 M 6). F, Minicythere argentinensis Bertels \& Martínez, 1997 (x649), external view LV (MiUNS 313, M 305). G, Cushmanidea echevarriae Bertels \& Martínez, 1997 (x588), external view RV (MiUNS 312, M 301). H, Callistocythere litoralensis (Rossi de García) 1966 (x868), external view LV (MiUNS 329 (1), M 301). I, Leptocythere darwini Whatley et al., 1997 (x682), female LV external view (MiUNS (1), M 279); J, Leptocythere darwini Whatley et al., 1997 (x582), male RV external view (MiUNS 328(2), M 301). Scale bars $=30 \mu \mathrm{m}$. 


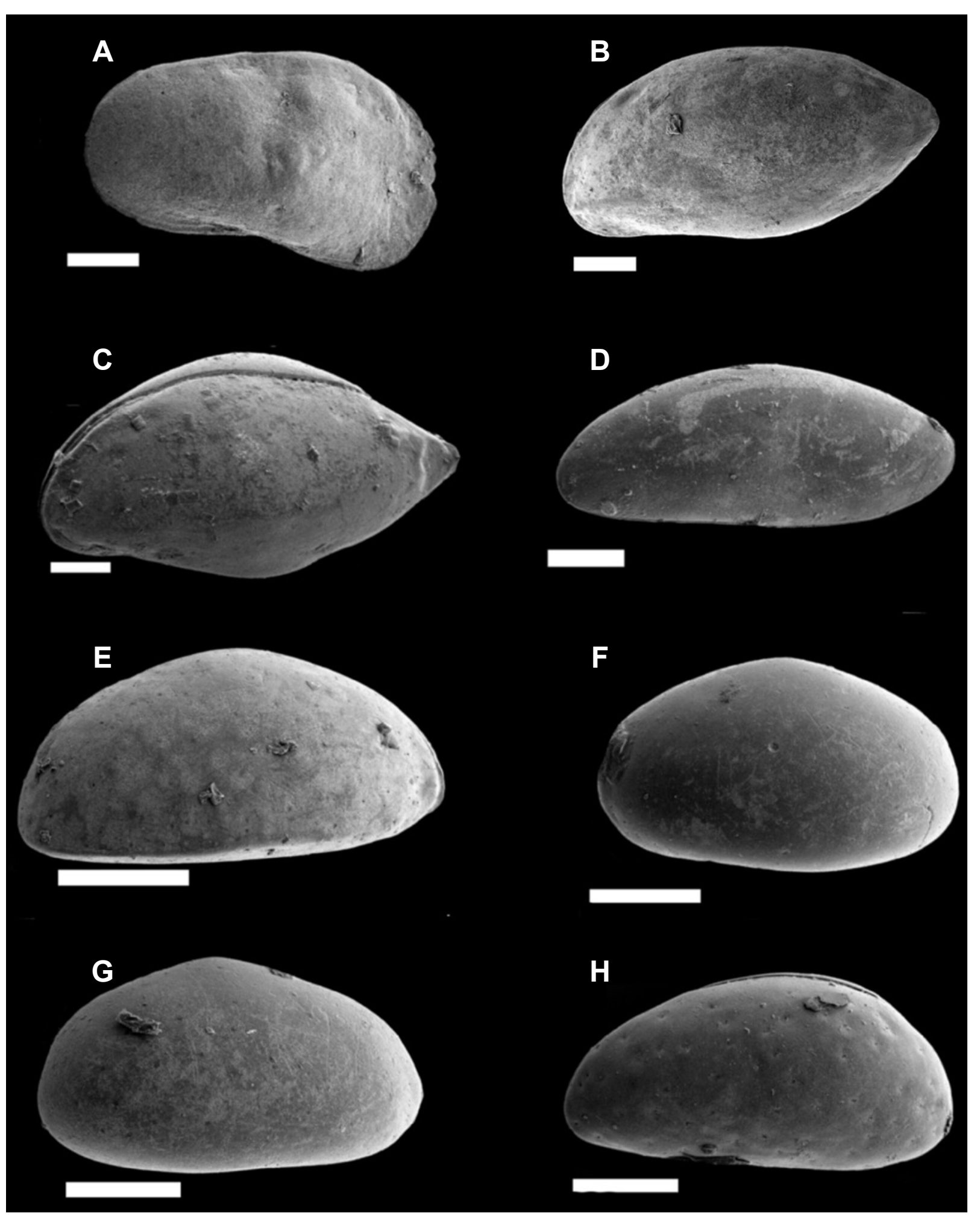

Figura 4. A, Limnocythere sp. (x518), vista externa VD hembra (MiUNS 332 (2), M 32). B-C, Pellucistoma elongata Whatley et al., 1998 B, (x636), vista externa VD (MiUNS 337, M 105); C, (x636), caparazón hembra vista externa VD (MiUNS 337, M 105). D, Cytherois sp. (x530), vista externa VI (MiUNS 338, M 2). E, Xestoleberis sp. (x856), vista externa VD (MiUNS 343, M 5); F, Semixestoleberis sp. (x943), vista externa VD (MiUNS 344, M 17); G, Semixestoleberis sp. 1 (x880), vista externa VI (MiUNS 345, M 10); H, Semixestoleberis debueni Hartmann, 1962 (x750), caparazón vista externa VI (MiUNS 346, M 287). Escalas $=30 \mu \mathrm{m}$.

Figure 4. A, Limnocythere sp. (x518), external view right female valve (MiUNS 332 (2), M 32). B-C, Pellucistoma elongata Whatley et al., 1998 B, (x636), RV external view (MiUNS 337, M 105); C, (x636), female carapace RV external view (MiUNS 337, M 105). D, Cytherois sp. (x530), LV external view (MiUNS 338, M 2). E, Xestoleberis sp. (x856), external view RV (MiUNS 343, M 5); F, Semixestoleberis sp. (x943), external view RV (MiUNS 344, M 17); G, Semixestoleberis sp. 1 (x880), LV external view (MiUNS 345, M 10); H, Semixestoleberis debueni Hartmann, 1962 (x750), LV shell external view (MiUNS 346, M 287). Scale bars $=30 \mu \mathrm{m}$. 
Cambios del nivel medio del mar durante el Holoceno en el estuario de Bahía Blanca

Con posterioridad al último máximo glaciar, el nivel medio del mar (NMM) ascendió desde profundidades mayores a los $100 \mathrm{~m}$, alcanzando las máximas alturas por encima del NMM actual a los cal. 6.900-6.000 años AP, para luego descender de manera aproximadamente paulatina hasta su posición actual (Cavalloto et al., 2004). Sin embargo sobre este comportamiento de mayor escala temporal, tanto en Brasil como en Argentina existen evidencias que indican la ocurrencia de oscilaciones negativas relativamente breves del NMM. Oscilaciones entre los cal. 2.700-2.330 años AP y entre los cal 4100-3750 años AP aproximadamente, fueron reconocidas en Brasil (Martin et al., 2003; entre otros), mientras que en el área de Bahía Blanca, Argentina, Gómez et al. (2005b, 2006) y Cusminsky et al. (2009) reconocen oscilaciones del NMM por debajo del actual para los cal. 7.300-6.600 años AP (A) y los cal. 2.700-2.330 años AP (C). Angulo et al. (2006), desestiman la ocurrencia de las oscilaciones a los 4.100-3.750 años AP y 2.700-2.330 años AP en Brasil aunque en virtud de la información que estos autores presentan ello no sería posible debido a que la incertidumbre temporal de las edades calibradas presentadas (700-1.030 años), supera la magnitud máxima probable de las oscilaciones propuestas. El estudio micropaleontológico y sedimentológico del testigo analizado en el presente trabajo permitió corroborar lo hipotetizado por Gómez et al. (2006) a partir de información presentada por Cavallotto et al. (2004), quién sugirió que la primera oscilación del NMM detectada en Brasil (aproximadamente a los cal 5.200 años

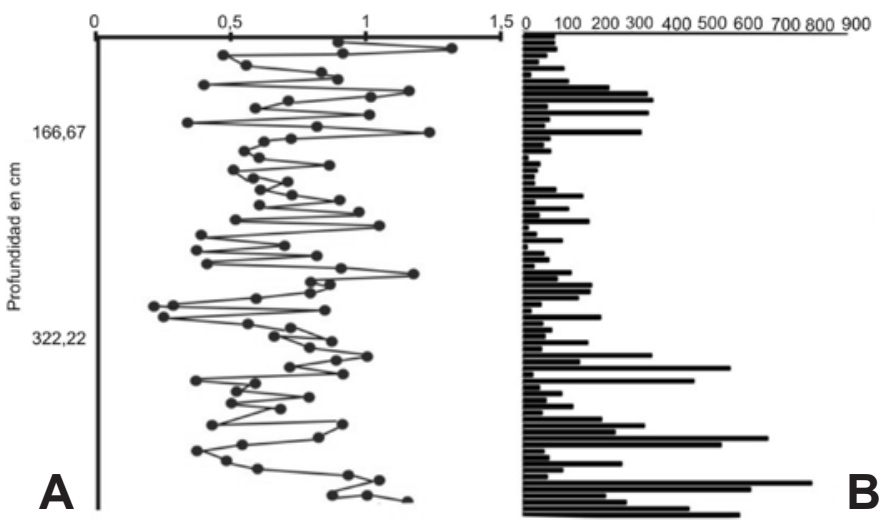

Figura 5. A, índice de diversidad de Shannon-Weaver; B, densidad (número de individuos en $100 \mathrm{~g}$ de sedimento).

Figure 5. A, diversity index Shannon-Weaver; B, density (number of individuals in $100 \mathrm{~g}$ of sediment).

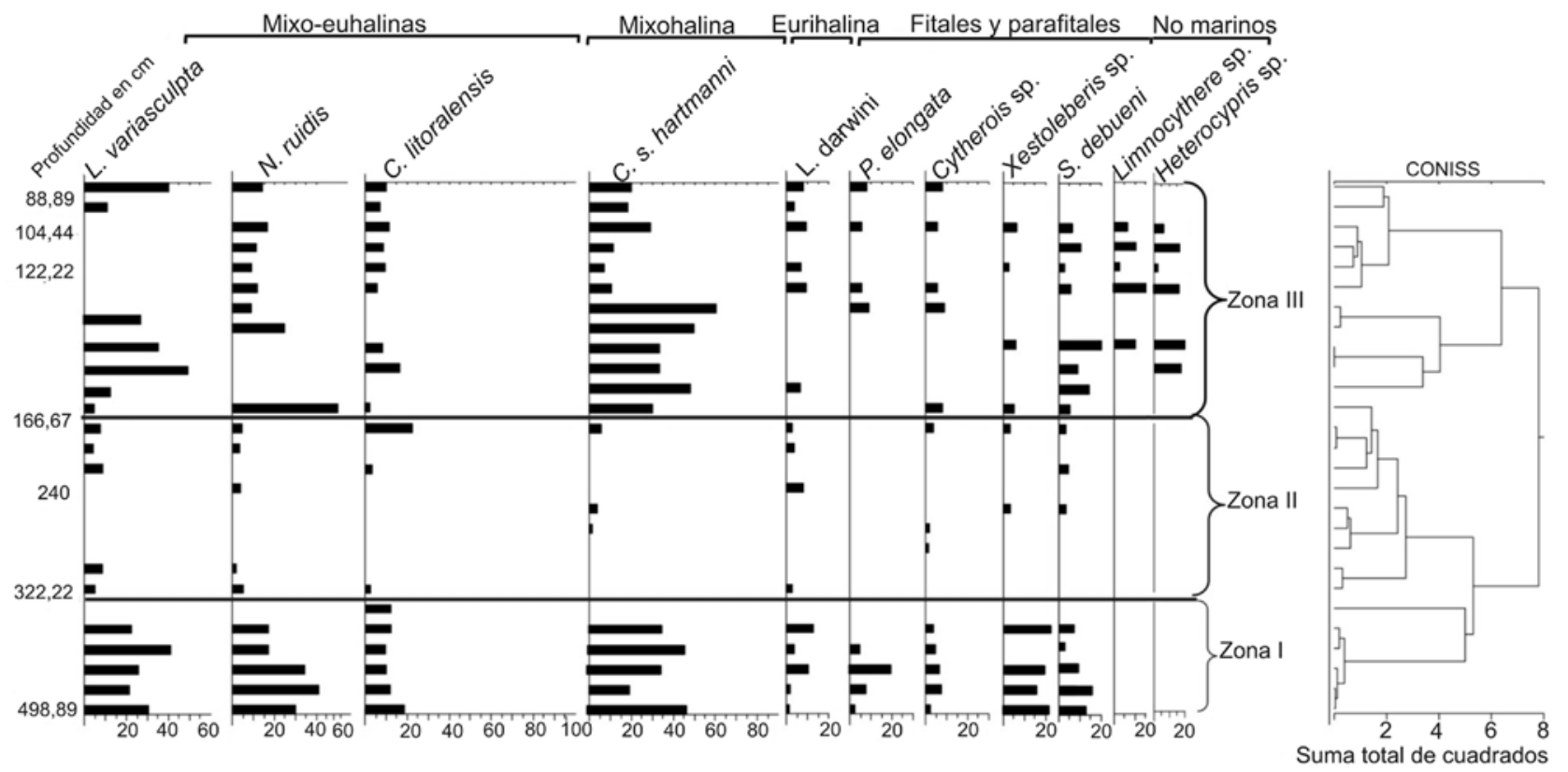

Figura 6. Diagrama de frecuencias de ostrácodos bentónicos autóctonos con una abundancia mayor al $5 \%$ (porcentaje). Incluye cluster analysis mostrando las zonaciones reconocidas por asociaciones de especies.

Figure 6. Frequency diagram of autochthonous benthic ostracods with percentage more abundant than $5 \%$. It includes cluster analysis showing recognized zonation by species association. 
AP) también habría tenido lugar en la Argentina, aunque ésta habría sido de menor magnitud que las otras oscilaciones detectadas, pero coincidiendo con valores extremos mínimos de gran variabilidad en la irradiación solar que ocurren con una periodicidad de aproximadamente unos 2.400 años (Gómez, 2004; Gómez et al., 2005a). Existe una fuerte controversia relacionada con la posición del nivel medio del mar en torno al Holoceno medio (Martin et al., 2003; Angulo et al., 2006, entre otros). Sin embargo, Gómez et al. (2011) y Kihn (2015) han demostrado la posibilidad de ocurrencia de oscilaciones rápidas del nivel medio del mar, probablemente relacionados con una alta variabilidad en la irradiación solar que se produjo entre cal. 4.500 y 5.500 años AP. Marquez et al. (2016) determinan un máximo de influencia marina en Las Brusquitas para los cal. 6.300-6.200 años AP y luego a los cal. 4.400-4.200 años AP detectan una leve y esporádica influencia marina; si bien Las Brusquitas y el estuario de Bahía Blanca se encuentran ubicados en la Provincia de Buenos Aires ambos ambientes presentan características sedimentológicas diferentes que dificultan la comparación entre los sitios.

\section{CONCLUSIONES}

El estudio de sedimentos de subambientes actuales permitió establecer que en las planicies de marea las especies Cyprideis salebrosa hartmanni y Callistocythere litoralensis son dominantes; en las marismas vegetadas con Spartina densiflora, C. litoralensis es la especie dominante con poblaciones muy desarrolladas; mientras que Neocytherideis ruidis y Loxocythere variasculpta sólo se hallan en la marisma baja vegetada por $S$. alterniflora. En base a los resultados obtenidos del análisis de muestras actuales se pudo determinar con mayor grado de confianza los cambios ambientales producidos y registrados en los sedimentos del Holoceno.

El estudio sistemático detallado de la ostracofauna presente en los distintos niveles del testigo TB permitió diferenciar, en base a las especies autóctonas registradas, los paleoambientes en los cuales se depositó el sedimento. En la base del testigo $(498,89-322,22 \mathrm{~cm})$ con una edad convencional de 5.090 \pm 40 años AP (cal. 5.662-5.907 años AP) los sedimentos fueron depositados en un ambiente intermareal con marismas desarrolladas; en el sector medio $(322,22-166,67 \mathrm{~cm})$ con una edad convencional de $4.760 \pm 40$ años AP (cal. 5.322-5.583 años AP) la escases de ostrácodos y la gran cantidad de fragmentos de conchillas y gasterópodos transportados evidenciaron un aumento en la energía ambiental y en el tramo superior del testigo la presencia de especies mixo-halinas y continentales permitió determinar un ambiente intermareal con marismas desarrolladas y aporte de aguas continentales. El desarrollo de marismas en la base de este testigo ubicadas a $4,80 \mathrm{~m}$ por debajo del NMM actual y a una altura que en la actualidad correspondería a ambiente submareal permanente, sugiere que la ubicación del NMM fue levemente por debajo de la actual para los 5.090 \pm 40 años AP.

\section{AGRADECIMIENTOS}

Este estudio fue financiado por el proyecto PICT 2007RAICES 00109, proyecto UTN 25/8035-UTI989, PICT 2008-1878, Beca CONICET doctoral. Los resultados son parte de la tesis doctoral de R. Kihn. Queremos agradecer las sugerencias de los revisores el C.T. Bergue y anónimo que han contribuido en forma significativa a mejorar el trabajo.

\section{REFERENCIAS}

Angulo, R.J.; Lessa, G. \& de Souza, M.C. 2006. A critical review of mid to late Holocene sea-level fluctuations on the eastern Brazilian coastline. Quaternary Science Reviews, 25:486-506. doi:10.1016/j.quascirev.2005.03.008

Beigt, D. 2007. Balance energético de las planicies de marea del estuario de Bahía Blanca. Universidad Nacional del Sur, Tesis doctoral, $220 \mathrm{p}$.

Bertels, A. \& Martínez, D.E. 1990. Quaternary ostracodes of continental and transitional littoral-shallow marine environments. Courier Forschchungs Institut Senckenberg, 123:141-159.

Bertels, A. \& Martínez, D.E. 1997. Ostrácodos holocenos de la desembocadura del arroyo Napostá Grande, sur de la provincia de Buenos Aires, Argentina. Revista Española de Micropaleontología, 29:20-69.

Bertels-Psotka, A. \& Laprida, C. 1998a. Ostrácodos (Arthropoda, Crustacea) Holocenos del Miembro Canal 18 (Formación Las Escobas), provincia de Buenos Aires. Revista Española de Micropaleontología, 30:129-137.

Bertels-Psotka, A. \& Laprida, C. 1998b. Ostrácodos (Arthropoda, Crustacea) del Miembro Cerro de la Gloria, Formación Las Escobas (Holoceno), provincia de Buenos Aires, República Argentina. Revista Española de Micropaleontología, 30:103-127.

Bertels-Psotka, A. \& Laprida, C. 1998c. Ostrácodos (Arthropoda, Crustacea) de la Formación Las Escobas (Holoceno), Cuenca del Salado, República Argentina. Ameghiniana, 35:81-86.

Bertels-Psotka, A. \& Laprida, C. 1998d. Paleoambientes holocenos del nordeste de la provincia de Buenos Aires. Ameghiniana, 35:151-162.

Bertels-Psotka, A. \& Martínez, D.E. 1999. Frenguellicythere argentinensis $\mathrm{n}$. gen. and n. sp. from Holocene deposits of estuary of Bahía Blanca, Buenos Aires, Argentina. Micropaleontology, 45:394-398. doi:10.2307/1486121

Boomer, I.; Home, D.J. \& Slipper, I.J. 2003. The use of ostracods in palaeoenvironmental studies, or what can you do with and ostracod shell? In: E.L. Park \& A.J. Smith (eds.) Bridging the gap. Trends in the ostracode Biollogical and Geological Sciences, Tuscaloosa, Paleontological Society, p. 153-179 (Special Papers 9).

Buzas, M.A. \& Gibson, T.G. 1969. Species diversity: Benthonic Foraminifera in Western North Atlantic. Science, 163:72-75. doi:10.1126/science.163.3862.72

Calvo Marcilese, L. \& Pratolongo, P. 2009. Foraminíferos de marismas y llanuras de marea del estuario de Bahía Blanca, Argentina: Distribución e implicaciones ambientales. Revista Española de Micropaleontología, 41:315-332.

Carbonel, P. 1980. Les ostracodes et leur interet deans la definition des ecosystemes estuariens et de plateforme continentale essais d'application a des domaines anciens. Institute de Geologie du Bassin d'Aquitaine, Université de Bordeaux, Tesis doctoral, $350 \mathrm{p}$. 
Cavallotto, J.L.; Violante, R.A. \& Parker, G. 2004. Sea-level fluctuations during the last 8600 years in the de la Plata River (Argentina). Quaternary International, 114:155-165. doi:10.1016/S1040-6182(03)00050-8

Coimbra, J.C.; Carreno, A.L. \& Ferron, F.A. 1994. Holocene podocopida Ostracoda from Sepetiba Bay, Brazil- Some Dominant taxa. Pesquisas em Geociências, 21:90-99.

Coimbra, J.C.; Carreno, A.L.; Geraque, E.A. \& Eichler, B.B. 2007. Ostracodes (Crustacea) from Cananeia.Iguape estuarine/ lagoon system and geographical distribution of the mixohaline assemblages in southern and sourtheastern Brazil. Iheringia. Serie Zoologia, 97:273-279. doi:10.1590/s0073-47212007000300010

Cronin, T. 1979. Late Pleistocene marginal marine ostracodes from the Southeastern Atlantic Coastal plain and their paleoenvironmental implications. Géographie physique et Quaternaire, 33:121-173. doi:10.7202/1000066ar

Cusminsky, G.C.; Bernasconi, E. \& Calvo-Marcilese L. 2009. Holocene benthic foraminifera from Bahía Blanca estuary: a review and update of systematic and palaeoenvironmental aspects. The Holocene, 19:1221-1231. doi: 10.1177/0959683609345085

Ferrero, L. 2006. Micropaleontología y Paleoecología del Cuaternario del sudeste de la provincia de Buenos Aires. Facultad de Ciencias Exactas y Naturales, Universidad Nacional de Mar del Plata, Tesis doctoral, 373 p.

Ferrero, L. 2009. Foraminíferos y ostrácodos del Pleistoceno tardío (Mar Chiquita, provincia de Buenos Aires, Argentina). Ameghiniana, 46:637-656.

Freije, R.H.; Zavatti, J.; Gayoso, A.M. \& Asteasuain, R. 1981. Producción primaria, pigmentos y fitoplancton del estuario de Bahía Blanca. Contribución Científica Instituto Argentino de Oceanografia, 46:1-12.

Gibson, T.G. \& Buzas, M.A. 1973. Species diversity: patterns in Modern and Miocene Foraminifera of the eastern margin of North America. Geological Society of America Bulletin, 84:217238. doi:10.1130/0016-7606(1973)84<217:SDPIMA>2.0.CO;2

Ginsberg, S.S. \& Perillo, G.M. 1990. Channel Bank Recession in the Bahía Blanca estuary, Argentina. Journal of Coastal Research, 6:999-1009.

Gómez, E.A. 2004. Sea Level Oscillations Related to Past Global Climatic Changes. Inter-American Institute for Global Change Research, News Letter, 36:11-13.

Gómez, E.A; Borel, C.M.; Aguirre, M.L., \& Martínez, D.E. 2008. Radiocarbon reservoir ages and hardwater effect for the northeastern coastal waters of Argentina. Radiocarbon, 50:119-129.

Gómez, E.A.; Kihn, R.G.; Martínez, D.E.; Borel, C.M.; Carbonella, J.C.; Raniolo, L.A.; Cuesta, A.C.; Lara, R.J. \& Grecco, L.E. 2011. Oscilación del nivel medio del mar por debajo de su posición actual durante el Holoceno medio. In: CONGRESSO LATINO-AMERICANO DE CIÊNCIAS DO MAR, 14, 2011. CD-rom de Resumos, Camboriú.

Gómez, E.A.; Martínez, D.E.; Borel, C.M.; Guerstein, G.R. \& Cusminsky, G.C. 2005a. Submarine evidence of Holocene sealevel fluctuations in the Bahía Blanca Estuary, Argentina. Journal of South American Earth Sciences, 20:139-155. doi:10.1016/j. jsames.2005.06.011

Gómez, E.A.; Martínez, D.; Borel, C.M.; Guerstein, G.R. \& Cusminsky, G.C. 2006. Negative sea-level oscillation at the Bahía Blanca Estuary related to a ca. 2650 yr BP global climatic change. Journal of Coastal Research, 39:181-185.

Gómez, E.A.; Martínez, D.E. \& Cusminsky, G.C. 2005b. A cycle in solar irradiance as trigger of abrupt Holocene global climatic changes and negative sea level oscillations. In: JOINT MEETING
OF INTERNATIONAL GEOSCIENCE PROGRAMME 490: HOLOCENE ENVIRONMENTAL CATASTROPHES IN SOUTH AMERICA: FROM THE LOWLANDS TO THE ANDES, 3, 2005. Abstracts, Cordoba, p. 25-26.

Grimm, E.C. 1991. Tilia software. Springfield, Illinois State Museum.

Hiltermann, H. 1985. Zur Methodik, Brackwasser-Sedimente zu erkennen und zu unterscheiden. Facies, 12:243-252. doi:10.1007/BF02536981

Hogg, A.G. et al. 2013. SHCal13 Southern Hemisfere calibration, 0-50000 years cal BP. Radiocarbon, 55:1889-1903. doi:10.2458/ azu_js_rc.55.16783

Horne, D.J.; Cohen, A. \& Martens, K. 2002. Taxonomy, Morphology and Biology of Quaternary and Living Ostracoda. In: J.A. Holmes \& A.R Chivas (eds.) The Ostracoda: Aplications in Quaternary Research, Washington, American Geophysical Union, p. 5-36 (Geophysical Monograph 131). doi:10.1029/131GM02

Hughen, K.A. et al. 2004. Marine04 marine radiocarbon age calibration, 0-26 cal kyr BP. Radiocarbon, 46:1059-1086.

Kidwell, S.M.; Fürsich, F.T. \& Aigner, T.H. 1986. Conceptual framework for the analysis and classification of fossil concentrations. Palaios, 1:228-238. doi:10.2307/3514687

Kihn, R.G. 2015. Estudio de las asociaciones de ostrácodos (Arthropoda, Crustacea) en depósitos transicionales y marinos como indicadoras de paleoambientes del Holoceno. Universidad Nacional del Sur, Tesis doctoral, $236 \mathrm{p}$.

Laprida, C. 2006. Ostracodos recientes de la llanura pampeana, Buenos Aires, Argentina: ecologia e implicancias paleolimnologicas. Ameghiniana, 43:181-204.

Laprida, C. \& Ballent, S. 2007. Ostracoda. In: H.H. Camacho (ed.) Invertebrados Fósiles, v. 2, Fundación de Historia Natural Félix de Azara y Universidad CAECE, p. 599-624.

Laprida, C. \& Valero-Garcés, B. 2009. Cambios ambientales de épocas históricas en la pampa bonaerense en base a ostrácodos: historia hidrológica de la laguna de Chascomús. Ameghiniana, 46:95-111.

Marquez, M.; Ferrero, L. \& Cusminsky, G. 2016. Holocene palaeoenvironmental evolution of the pampa coastal plain (Argentina) based on calcareous microfossils. Revista Brasileira de Paleontologia, 19:25-40. doi:10.4072/rbp.2016.1.03

Martin, L.; Dominguez, J.M.L. \& Bittencourt, A.C.S.P. 2003. Fluctuating Holocene Sea Levels in Eastern and Southeastern Brazil: Evidence from Multiple Fossil and Geometric Indicators. Journal of Coastal Research, 19:101:124.

Martínez, D.E. 2005. Asociaciones de ostrácodos modernos del estuario de Bahía Blanca, Buenos Aires, Argentina. Ameghiniana, 42:669-684.

Moore, R. 1961. Treatise on Invertebrate Paleontology. Part Q. Arthropoda 3: Crustacea, Ostracoda. New York, Geological Society of America, $442 \mathrm{p}$.

Ornellas, L.P. \& Würdig, N. 1983. Cyprideis salebrosa hatmanni Ramírez, F., 1967, a new subspecies from Brazil and Argentina. Pesquisas, 15:94-112.

Piccolo, M.C.; Perillo G. \& Arango, J.M. 1987. Hidrografía del estuario de Bahía Blanca, Argentina. Revista Geofísica, 26:75-89.

Shannon, C.E. \& Weaver, W. 1949. The Mathematical Theory of Communication. Urbana, University of Illinois Press, 125 p.

Verettoni, H. 1961. Las asociaciones halófilas de partido de Bahía Blanca. Bahía Blanca, Comisíon Ejecutiva del 150 Aniversario de la Revolucíon de Mayo, 150 p. 
Verettoni, H. \& Aramayo, E. 1976. Las comunidades vegetales de la región de Bahía Blanca. Bahía Blanca, Harris \& Cia SRL, 175 p.

Whatley, R.C.; Chadwick, J.; Coxill, D. \& Toy, N. 1987. New genera and species of Cytheracean Ostracoda from the S. W Atlantic. Journal of Micropaleontology, 6:1-12. doi:10.1144/jm.6.2.1

Whatley, R.C.; Chadwick, J.; Coxill, D. \& Toy, N. 1988. The ostracod family Cytheruridae from the Antartic and South West Atlantic. Revista Española de Micropaleontología, 20:171-203.
Whatley, R.C. \& Cusminsky, G.C., 1995. Quaternary lacustrine Ostracoda from northern Patagonia, Argentina. In: J. Riha (ed.) Ostracoda and Biostratigraphy, A.A.Balkema, p. 303-310.

Whatley, R.C.\& Moguilevsky, A. 1975. The family Leptocytheridae in Argentina waters. Bulletin of American Paleontology, 65:502-517.

Received in May, 2016; accepted in August, 2016. 